Dr Freeman alleges that my present work stems mainly from the theories of Dr R. D. Laing. This is not the case. Although I hold Dr Laing in respect. I have not worked with him for many years. My current work, particularly with the Arbours Association, which sponsors a Crisis Centre and residential communities, derives from many sources including Dr Maxwell Jones and his colleagues on therapeutic communities, Drs Gerald Caplan, Donald Langsley, R. D. Scott and their colleagues on crisis intervention and current thinking on the psychoanalytic treatment of the borderline and psychotic patient.

That schizophrenic patients respond to the unconscious mental processes of their therapists, and especially to their interest and enthusiasm, is indeed well known and has been described by psychoanalysts including Drs John Rosen and Harold Searles.

I am afraid that Dr Freeman has misstated my analysis of the work of the Arbours Crisis Centre. Over the four-year period, January 1973 to February 1977, almost 150 people were resident at the Centre. Many hundreds more were helped by telephone interventions (no visit required) or through individual and family meetings held at their homes or at the Centre.

29 Oval Road,

JO8EPH H. BERKE

London NW1 7EA

\section{NURSE THERAPISTS}

DeAr Sir,

With growing concern I have read the correspondence in your columns about the training of nursetherapists. As one of the few psychiatric nurses currently engaged in full-time clinical research in psychiatric nursing, I find it sad that the very important issue of what roles psychiatric nurses currently have, and what they ought, in future, to have, has been obscured by this correspondence. Several points ought to be made.

Whilst Professor Goldberg's review (Journal, September 1977, 131, 320) of Dr Mark's research used an analogy which was, perhaps, a little unfortunate, in comparing psychiatric nurses with lorry drivers, there is no doubt that nurses do have their own role within the health system. If other members of the team such as psychiatrists and psychologists see psychiatric nurses merely as a convenient source of extra personnel to be used to supplement the gaps in their services then this is also unfortunate, since it suggests that psychiatric nurses currently have nothing in particular to do, unless directed by other professionals.

It is also sad that the term 'nurse-therapist' has become almost exclusively attached to those who have completed Dr Mark's behaviourally oriented course. Many psychiatric nurses would consider themselves therapists, but would make their therapeutic interventions from other models of psychiatry.

Important, too, are developments in nursing. The Department of Nursing here is committed to the development of planned and systematic nursing care for all patients. Developing this approach to nursing demands a high level of knowledge in the behavioural and biological sciences. If psychiatric nurses are to provide this type of care for patients, then they are likely to find their time is fully occupied.

One would hope that psychiatric nurses will have the courage to develop their own, highly important, professional role and to let psychiatrists and psychologists find their assistants from other sources. In this way the most important member of the team-the patient-will be assured of a high standard of service from all professionals in the health care team. Helen Macilwaine

University of Manchester,

Department of Nursing,

Stopford Building,

Oxford Road, Manchester M13 9PT

This correspondence is now closed.-Editor.

DeAr Sir,

\section{WARD ROUNDS}

As suggested by Dr Baxter in the May 1978 issue of the Fournal (132, 526) I would like to report a method for reviewing and meeting patients which has been worked out by my unit in a District General Hospital setting. The team as a whole reviews the case of each patient and then, as a group, we meet with the patients concerned sharing with them any suggestions and decisions of immediate concern to them such as changes in medication or weekend leave, as well as matters of deeper significance if appropriate. This meeting often leads on to become a community meeting with its wider implications and is naturally followed by a feedback when the staff meet together without the patients. We may then revise earlier decisions in the light of discussion at the meeting with patients. The patients do of course, have other opportunities for meeting both medical and non-medical members of staff in individual or group sessions.

It is my impression that our format for a ward round allows a multi-disciplinary approach to patient management with the participation of patients, but it should be seen in the context in which it occurs, namely, a setting based on therapeutic community principles. Although our format has obvious disadvantages we feel that it offers a better 
setting for a multi-disciplinary review of patients than does a traditional ward round when individual patients are interviewed by a whole team. Firstly it avoids the sort of intimidation that an individual patient might feel when confronted by several members of staff en masse-'much worse than vivas' according to your correspondent (Joumal, January $1978,132,112$ ). The group setting seems to enable patients to question staff in a way which is seldom seen in the traditional ward round. We feel that this approach is conducive to the sharing of decision- 1 making with patients and therefore therapeutic in itself; patients seem to be able to feel their way towards participating in making decisions without arousing too much staff anxiety, or insecurity in themselves. Secondly our type of approach is a way of enforcing rather than undermining the therapeutic momentum of self-help among patients-a force which I believe is potentially a highly effective tool in the treatment of psychiatric illness. Finally our ward round provides a suitable framework in which members of staff can examine their own motives and wishes during decision-making, thereby improving the quality of decisions.

Department of Psychiatry, Suman Fernando

Enfield District Hospital, Chase Wing, Enfield, Middx.

\section{CHRONIC ANXIETY IN ABORIGINALS}

Dear Sir,

Morice (1978), in an interesting approach to psychiatric illness among Australian Pintubi aboriginals, infers the existence of anxiety and depression from the presence of words in their vocabulary describing these affects. From his glossary it is apparent that these people include an inner feeling state, autonomic correlates, 'varying degrees of severity and differing provoking situations' as correlates. These observations are totally in accord with our experience of psychiatric symptoms in western desert aboriginals (Jones, 1972; Jones and Horne, 1973), but we called these phenomena 'fear'. We found very few examples of this state persisted for more than a few hours, although we drew our data from a survey of approximately 2,300 persons including some Pintubi. It will be noted that $\mathrm{Dr}$ Morice's glossary only includes descriptions of acute states. We concluded that chronic anxiety (which perhaps inadvisedly we called 'overt anxiety') was rare. His paper seems to give support to this proposition rather than refutation of it.

If these observations can be further supported they could be of value in highlighting a transcultural difference; presumably of developmental origin. Chronic states which might be anxiety equivalents do exist, for example hypochondriacal syndromes are common but these are usually quite free from autonomic correlates of anxiety.

Whether the proposition that chronic anxiety is infrequent in tribal aboriginals can now be established is, unfortunately, less than certain: even the most distant aboriginal groups are taking on white Australian ways and mores while losing their own culture. If the difference is real and culturally determined, one might expect more cases of anxiety to appear. If despite these social changes chronic overt anxiety remains infrequent, the question would be even more worthwhile investigating since it may indicate a biological difference. This difference in anxiety and the infrequent occurrence of homosexuality were the major findings in our studies. Most other psychiatric states were seen in recognizable form (Jones and Horne, 1972).

University of Melbourne, IVOR JONES

Department of Psychiatry, St Vincent's Hospital,

Fitzroy 3065,

Victoria, Australia

\section{References}

JONEs, I. H. \& HORNE, D. J. (1972) Diagnosis of psychiatric disorders among tribal Aboriginals. Medical foumal of Australia, 1, 345-9.

(1972) Psychiatric disorders of Aborigines of the Australian Western desert (ii). Social Science and Medicine, 6, 263-7.

— \& Horne, D. J. DE L. (1973) Psychiatric disorders among Aborigines of the Australian Western desert. Social Science and Medicine, 7, 219-28.

- \& Robinson, I. (1977) Severe illness with anxiety following a reputed magical act on an Australian Aboriginal. Medical Journal of Australia, 2, 93-6.

Morice, R. (1978) Psychiatric diagnosis in a transcultural setting. British Foumal of Psychiatry, 132, 87-95.

\section{BIOAGTIVE AMINES}

Dear Sir,

Research into central nervous system functions of different bioactive amines has yet to come up with some unifying hypotheses. I wish to propose an hypothesis that seeks to explain an apparent coincidence that substances released in skin injury (e.g. histamine) and vascular injury (serotonin) are also suspected of being regulator substances in the C.N.S.

I believe future research will demonstrate a general principle that, regardless of species or the particular amines released, those amine substances released from 